\title{
Strongly nonlinear dynamics of ferroelectric liquid crystals
}

\author{
W. Jeżewski ${ }^{a}$, I. Śliwa, and W. Kuczyński \\ Institute of Molecular Physics, Polish Academy of Sciences, Smoluchowskiego 17, Poznań, Poland
}

Received 28 August 2012 and Received in final form 30 November 2012

Published online: 15 January 2013

(c) The Author(s) 2013. This article is published with open access at Springerlink.com

\begin{abstract}
Molecular reorientation processes induced in thin ferroelectric liquid crystal systems by strong alternating external electric fields are studied both by solving numerically the equation of reorientation motion of molecules and by measuring the electro-optic response of thin samples. It is shown that the occurrence of a wide band in nonlinear response spectra above the Goldstone-mode frequency is a consequence of complex partially uncorrelated molecular reorientations enforced within smectic layers by sufficiently high fields of high enough frequencies. Such nonlinear reorientational motions of molecules are argued to have a character of weakly chaotic long-lasting transients, related to almost periodic modulations of the amplitude of rotational oscillations performed by molecules with the field frequency. These modulations have been numerically proved to proceed with lower frequencies than the field frequency and with space-dependent depths of temporal changes. The occurrence of the modulations has experimentally been confirmed by registering distinct contributions to electro-optic response spectra at frequencies less than the running frequency of the applied electric field.
\end{abstract}

\section{Introduction}

Nonlinear effects of molecular reorientations in liquid crystals (LCs) have intensively been studied under excitations mediated both by high electric field and intense light [1-8]. Spatiotemporal dynamic processes underlying these effects play an important role not only in basic research but also in technological applications. In particular, the electro-optic switching process [9], which is strongly nonlinear in nature, underpins the operations of modern display, light modulators, and switch devices. To investigate nonlinear effects in LCs, various methods have been developed, most of them, however, in the context of the nonlinear dielectric spectroscopy $[10-20]$. The dielectric response of smectic LCs on strong electric fields has been analyzed by determining higher harmonic components of the dielectric permittivity spectrum $\varepsilon(\omega)$ with $\omega=2 \pi f$, where $f$ is the frequency of an applied electric field. It has theoretically been shown that third-order and fifth-order harmonic contributions to $\varepsilon(\omega)$ involve only one characteristic time, i.e., that they are determined by one relaxation time, equal to the linear relaxation time $[5,11-14,16,17,19]$. However, experimental results obtained for $\mathrm{Sm} C$ systems with the helical superstructure suggest that the thirdorder and fifth-order relaxation times, characterizing, respectively, the third-order and fifth-order harmonic components of $\varepsilon(\omega)$, are different from the linear relaxation

\footnotetext{
a e-mail: jezewski@ifmpan.poznan.pl
}

time $[17,19]$, although the single time scale approach appears to be justified for some cases [11-14,17]. Clearly, one can expect that such a simple single time scale description of the nonlinear response of LCs, based on representing the azimuthal angle of molecules as a sum of fundamental and higher harmonics $[5,19]$, will be adequate for liquid crystalline materials that are rather stiff, i.e., for LC substances that display large elastic constants, and/or for applied electric fields which are not too strong. In such cases, the theoretical relation for fundamental contribution to nonlinear response spectra has a form of the Debye type, similarly as in the case of the linear response spectra $[16,17]$. In agreement with the theoretical prediction, the simple Debye form (or slightly broadened) of fundamental components of nonlinear spectra, related to collective molecular reorientation processes, have been reproduced experimentally [17].

The fundamental contribution to the response spectra of surface stabilized ferroelectric liquid crystal (SSFLC) systems, [21] experimentally determined for sufficiently strong fields, displays, however, much more complex form than the simple Debye type or somewhat broadened Debye type [22]. In addition to considerable influence on the fundamental harmonics of $\varepsilon(\omega)$ due to collective azimuthal reorientations of molecules within smectic layers, sufficiently strong fields induce high-frequency, relatively broad band which can even be separated from lowerfrequency band corresponding to collective processes. The appearance of the high-frequency band in the response 
spectra of SSFLCs can be interpreted as a consequence of decorrelation or partial decorrelation of molecular reorientations [22-24]. Such a complex molecular excitations can in turn be considered as resulting from an interplay between anchoring of molecules at plates confining samples and the action of strong enough electric field. It is thus clear that a proper description of complex dynamics of SSFLCs under very strong fields requires other approach than that used to determine nonlinear response spectra through seeking a solution of the motion equation for the azimuthal reorientations of molecules in a harmonic expansion. This follows from the mere fact that the method based on the harmonic expansion of the azimuthal angle does not account for the high-frequency band in the fundamental component of the response spectra. Naturally, the nonlinear motion equation describing azimuthal reorientations of molecules possesses solutions of different types, which apply in different regimes of amplitudes and frequencies of the external electric field. In particular, it has been shown that a motion equation involving depolarization interactions has a solution in the form of solitary waves [22]. However, solitons can be excited in thin samples only within a rather narrow range of field frequencies and, therefore, their propagation cannot be responsible for emerging a broad high-frequency band in the response spectra.

In this paper, the complex dynamics induced by strong alternating electric fields in SSFLC systems with the vertical chevron structure is studied both theoretically and experimentally. To analyze nonlinear effects of azimuthal reorientations of molecules, a usual dynamic field equation expressing the balance of moments acting on molecules is applied. However, solutions to this equation are found numerically, neither assuming a priori that they can be represented in harmonic expansion nor imposing any special functional form for their time dependence. Anchoring interactions at sample surfaces are taken into consideration implicitly by assuming that the azimuthal angle is space dependent, at least initially, i.e., when the external electric field is absent. The electric field is taken to be strong enough to dominate the anchoring interactions, so these interactions can be ignored as soon as the electric field is turned on. It is shown that even then the electric field cannot completely eliminate the inhomogeneity of the azimuthal angle for a class of initial space distributions of this angle (determined by the anchoring interactions). Furthermore, the systems are proved to display quasi-periodic processes in longer time scales than the scale of the oscillation period $1 / f$ of the electric field. An experimental evidence for the occurrence of such slow dynamic processes in strongly nonlinear SSFLC systems is given.

\section{Experimental background}

To analyze strongly nonlinear effects in LCs, the liquid crystalline mixtures Felix 15-100 and Felix 17-100 (Clariant) sandwitched between plates of thin cells were used as model systems. In thin cells (from a few to several mi- crons in thickness), these LCs form stable smectic layers of bookshelf or chevron structures at temperatures from a wide range [9]. Cells (Linkam), chosen to prepare measuring samples, possessed the same thickness $d=5 \mu \mathrm{m}$. They were constructed from glass plates coated with conducting, semitransparent material ITO (indium-tin-oxide), enabling light to pass through the samples. Experimental studies of nonlinear reorientations of molecules within smectic layers have been carried out by registering the response of planarly oriented samples to an external sinusoidally alternating electric field, with the use of the electro-optic method [25]. All measurements were performed at fixed temperature $T=30^{\circ} \mathrm{C}$, i.e., $47^{\circ} \mathrm{C}$ below the temperature of the transition of the studied LC materials from ferroelectric to paraelectric phase. At such thermal conditions, samples used in experiments formed stable chevroned layers, oriented nearly perpendicular to confining plates. Furthermore, the alignment of smectic layers remained rather stable throughout experiments performed at different applied alternating fields, as was evidenced by a large stability of sample textures. The amplitude of azimuthal reorientations remained relatively small (distinctly less than $\pi$ ), especially at intermediate and high field frequencies. Then, fixing the angle $\vartheta$ between the optic axis and the light polarization direction at the value $\vartheta=\pi / 8$, we were able to measure the coefficient of the light transmittance for values of the molecular azimuthal angle belonging to ranges (field-dependent) within which this coefficient changed monotonically with the azimuthal angle. Since all considerations presented below concern only the fundamental component of the response spectra, or more precisely their increments due to field-induced reorientations of molecules, the notation $\varepsilon(\omega) \equiv \varepsilon_{1}(\omega)$ is henceforth adopted for simplicity's sake. It should be pointed out that the fundamental components of the electro-optic spectra are denoted here by $\varepsilon(\omega)$ because dielectric and electro-optic responses of SSFLCs are usually consistent within a wide range of the field frequency, including the frequencies of special interest in this paper [26].

The electro-optic response spectrum measured for a high-voltage amplitude $U_{0}=20 \mathrm{~V}$ (rms) is shown in fig. 1 , where a spectrum determined for a weak-voltage amplitude $U_{0}=0.2 \mathrm{~V}$ is plotted for comparison. As is seen, the frequency dependence of the nonlinear spectrum evidently differs from the Debye-like form, typical for the linear spectra. It is striking that, for sufficiently high voltages, $\varepsilon(\omega)$ displays the existence of a rather wide high-frequency band. This band is distinctly visible in the diagram of $\varepsilon^{\prime \prime}(\omega)$ drawn in fig. 1 , above the frequency $f \approx 3 \mathrm{kHz}$. The appearance of a high-frequency band in nonlinear response spectra of SSFLCs can be considered as a consequence of a decrease of the correlation between molecular reorientations within smectic layers under strong electric fields. The resulting partially uncorrelated dynamic processes can certainly originate in a complex interplay between surface anchoring interactions, which tend to suppress molecular oscillatory rotations, especially effectively at sample surfaces, and the interactions of molecules with a strong electric field, which tends to rotate molecules with 


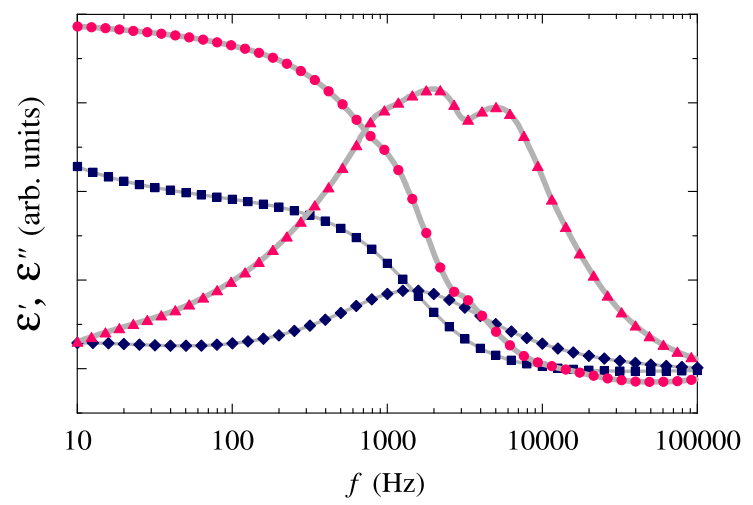

Fig. 1. Real and imaginary parts of nonlinear and linear electro-optic response spectra of Felix 15-100 LC, measured respectively for $U_{0}=20 \mathrm{~V}: \varepsilon^{\prime}(\omega)(\bullet), \varepsilon^{\prime \prime}(\omega)(\boldsymbol{\Delta})$ and for $U_{0}=0.2 \mathrm{~V}: \varepsilon^{\prime}(\omega)(\boldsymbol{\square}), \varepsilon^{\prime \prime}(\omega)(\boldsymbol{})$. The scale between the linear and nonlinear spectra is not respected.

a large amplitude. Essentially, the anchoring interactions act on molecules located at cell plates. However, owing to the existence of elastic couplings between molecules, these interactions influence also the orientation of molecules situated inside samples. As a result of the occurrence of the countervailing tendencies towards controlling the motion of molecules and/or as a consequence of the inhomogeneity of the electric field inside smectic layers, there can occur a space-dependent phase shift between rotational oscillations of molecules (located at different positions within a given smectic layer) and the field oscillations. Clearly, the dependence of the phase shift on the position of individual molecules is associated with the existence of different relaxation times of the system, and is thereby associated with the emergence of a rather broad frequency band in $\varepsilon(\omega)$. Another way in which the decorrelation of molecular orientational oscillations can manifest itself is a complex space and time dependence of the amplitude of these oscillations.

Essentially, one might suppose that the existence of different time scales in the studied LC mixtures would be a result of the appearance of individual dynamic processes associated with different compounds of these systems. This would be possible if molecular reorientations within smectic layers were completely desynchronized. However, such a desynchronization process might appear when elastic molecular interactions within smectic layers would be extremely small, but then this process should also appear at relatively weak fields. Thus, the occurrence of individual field-induced molecular reorientations connected with particular compounds of studied mixtures can be considered as improbable.

In order to examine the variation in the complex dynamic behavior of SSFLCs as the applied voltage grows, the Cole-Cole diagrams experimentally obtained for electro-optic response of a sample filled with Felix 17100 LC have been plotted in fig. 2 for a sequence of voltage amplitudes. This figure shows that there is no regular evolution of the response spectra when the voltage amplitude increases. It is seen that the Cole-Cole diagrams display

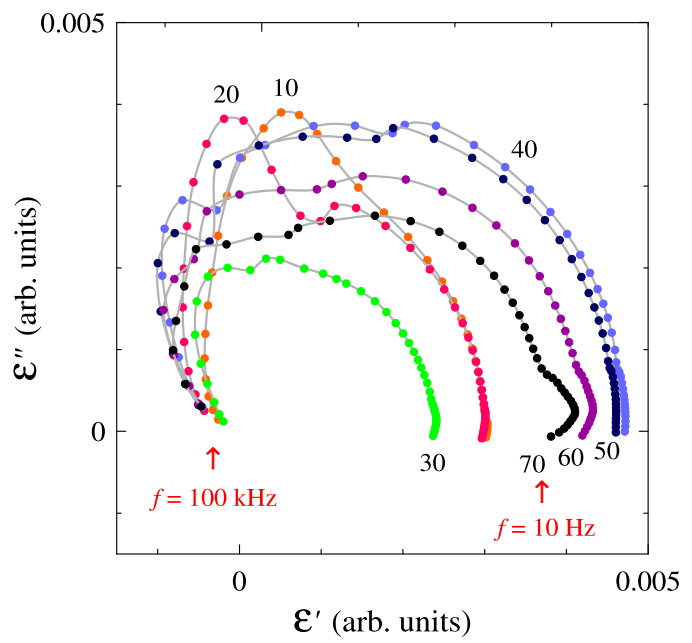

Fig. 2. Cole-Cole diagrams experimentally determined for Felix $17-100 \mathrm{LC}$ at $U_{0}=10,20, \ldots, 70 \mathrm{~V}$. The diagrams are marked by respective voltage amplitudes. The arrows indicate limiting measurement points, obtained for $f=10 \mathrm{~Hz}$ and $f=100 \mathrm{kHz}$.

a complex form even in the regime of low frequencies. In particular, the dependence of the diagrams on $U_{0}$ is not monotonous for a given (small) frequency. This can be considered as a simple consequence of the fact that relatively large contributions to the low-frequency part of the nonlinear response of SSFLCs not only come from collective reorientations of molecules but also from other dynamic processes, such as ionic currents and movements of walls of zig-zag defects in the regular chevron structure of SSFLCs [27-29]. As well known, the chevron structure spontaneously forms in SSFLCs, when their thickness is not very small. In general, this structure is transformed to the bookshelf or quasi-bookshelf structure under strong enough fields, and then the zig-zag defects are entirely or only partially destroyed. However, in cases of applied field oscillating with low frequencies, the defected chevron structure can temporarily rebuild when the field is equal to zero or is close to zero. Such rebuild processes can easily be observed when their characteristic times are faster than the period of the field alternation. Due to a large complexity of the low-frequency dynamic processes appearing in SSFLCs at strong fields, a theoretical description of these processes would be very difficult. Consequently, the analysis of the nonlinear behavior of SSFLCs is carried out here only for high external fields of rather high than low frequencies. Moreover, the irregular variation of the shape of the Cole-Cole diagram under changing $U_{0}$ is especially distinct for high frequencies. Clearly, the mere appearance of the high-frequency band in the fundamental component of the response spectra clearly indicates that the complex dynamics of SSFLCs in strong fields cannot be described by expressing the azimuthal angle of molecules in a form of the harmonic expansion. Below, an approach to analyze molecular reorientations induced by very strong fields is presented. 


\section{Numerical analysis of molecular rotational motions}

Field-induced reorientations of molecules in SSFLCs exhibiting vertical chevron geometry can be described by assuming that chevron slabs are oriented nearly perpendicular to sample plates and by assuming that the chevron structure is transformed to a quasi-bookshelf structure under the external electric field [9]. The effect of straightening of chevron smectic layers is especially distinct in the case of strong applied fields (constant or alternating) and then the smectic layers can be treated as being upright. Thus, the linear size of such uprighted smectic layers in the direction perpendicular to cell plates is equal to $d$. Spatial fluctuations of molecular orientations can be investigated separately within each of the smectic layers by determining the space dependence of the azimuthal angle $\phi$ between vectors of the local polarization and the external electric field [27-29]. Accordingly, the fluctuations of $\phi$ can be investigated only along one direction, say the $x$ direction, perpendicular to plates confining the sample. When an alternating electric field $E(t)=E_{0} \cos (\omega t)$ of the amplitude $E_{0}=U_{0} / d$ and the angular frequency $\omega$ is applied to sample plates, say in the $x$ direction, the spatiotemporal dependence of $\phi=\phi(x, t)$, where $0 \leqslant x \leqslant d$, can be found by solving the motion equation [9]

$$
K \frac{\partial^{2} \phi}{\partial x^{2}}-\gamma \frac{\partial \phi}{\partial t}=P_{S} E_{0} \sin \phi \cos \omega t,
$$

where $K$ is the elastic constant characterizing deformations inside smectic layers, $\gamma$ denotes the rotational viscosity, and $P_{S}$ is the spontaneous polarization. Inserting $x=\tilde{x} d$ and $t=\tilde{t} / \omega$ into eq. (1), one can express this equation in the following dimensionless form

$$
\frac{1}{2} c_{1} \frac{\partial^{2} \phi}{\partial \tilde{x}^{2}}-\frac{\partial \phi}{\partial \tilde{t}}=c_{2} \sin \phi \cos \tilde{t}
$$

with $0 \leqslant \tilde{x} \leqslant 1$ and with the parameters

$$
\begin{aligned}
& c_{1}=\frac{2 K}{\gamma \omega d^{2}}, \\
& c_{2}=\frac{P_{S} U_{0}}{\gamma \omega d} .
\end{aligned}
$$

The solution to the above motion equation is postulated here to have the nonuniform shape (cf., refs. [27-29]):

$$
\phi(\tilde{x}, \tilde{t})=a_{0}(\tilde{t})+a_{1}(\tilde{t}) \tilde{x}+a_{2}(\tilde{t}) \tilde{x}^{2},
$$

where the coefficients $a_{i}, i=0,1,2$, are space independent. Generally, these coefficients do not have all identical functional form. Initial values of $a_{i}$ (in the absence of the external electric field) are determined by appropriate boundary conditions, involving surface anchoring interactions [27-31]. After the electric field is switched on, the coefficients $a_{i}$ change according to electric field oscillations. This change can reveal different character depending on the frequency and the amplitude of the electric field. Clearly, the time dependence of $a_{1}$ and $a_{2}$ is associated with a temporal variation of the nonuniformity of $\phi$. For instance, special cases when both the coefficients $a_{1}$ and $a_{2}$ are decreasing functions of time and, conversely, when both $a_{1}$ and $a_{2}$ are increasing functions of time correspond respectively to global (for all $0 \leqslant \tilde{x} \leqslant 1$ ) fieldstimulated reduction and magnification of the nonuniformity level of $\phi$. The action of surface anchoring interactions is taken into account here only through boundary conditions at $E_{0}=0$. Obviously, in the presence of strong enough electric fields, molecular motions are almost entirely field-controlled, and then the anchoring effect due to surface interactions can be neglected. In this approximation, the azimuthal angle is determined without imposing on it any time-dependent boundary conditions.

Equations describing the time evolution of the coefficients $a_{i}, i=0,1,2$, can be obtained by substituting (5) into eq. (2). Consequently, one finds

$$
\begin{aligned}
\frac{\mathrm{d}}{\mathrm{d} \tilde{t}} a_{0} & =c_{1} a_{2}-c_{2} \cos \tilde{t} \sin a_{0}, \\
\frac{\mathrm{d}}{\mathrm{d} \tilde{t}} a_{1} & =-c_{2} \cos \tilde{t} a_{1} \cos a_{0}, \\
\frac{\mathrm{d}}{\mathrm{d} \tilde{t}} a_{2} & =-c_{2} \cos \tilde{t}\left(a_{2} \cos a_{0}-\frac{1}{2} a_{1}^{2} \sin a_{0}\right) .
\end{aligned}
$$

The role of nonlinearity parameters in the above set of equations is played by $c_{1}$ and $c_{2}$, which involve $\omega$ and $U_{0}$ (see eqs. (3) and (4)). To solve these equations, the Runge-Kutta-Gill numerical integration method [32] of fourth order has been used. Calculations have been performed for material constants determined at $T=30^{\circ} \mathrm{C}$ (for the LC mixture Felix 17-100 in a cell of thickness $d=5 \mu \mathrm{m})[22]: P_{S}=2.9 \times 10^{-4} \mathrm{C} \mathrm{m}^{-2}, \gamma=0.055 \mathrm{Pas}$, and $K=1.5 \times 10^{-9} \mathrm{~N}$. Values of the amplitude and frequency of the applied field have been taken to be $U_{0}=20$, $40,60 \mathrm{~V}$, and $f=3,4,5 \mathrm{kHz}$, respectively. Note that, at these values of $U_{0}$ and $f$, azimuthal rotations of molecules in the sample under study are rather weakly correlated (this is reflected in fig. 1).

Solutions to eqs. (6)-(8) have been derived in the form of trajectories generated in the space $\left(a_{0}, a_{1}, a_{2}\right)$ for discrete integer-valued time $t_{n}=t_{0}+n h$, where $t_{0}$ denotes time at which the electric field is switched on, $h>0$ is the sampling interval, and $n=0,1,2, \ldots$. More precisely, the use of the numerical integration procedure to eqs. (6)-(8) yields discrete flow maps, which can be iterated beginning with some initial values $a_{0}^{(0)}, a_{1}^{(0)}$, and $a_{2}^{(0)}$ (at $n=0$ ) of the coefficients $a_{0}, a_{1}$, and $a_{2}$, respectively. Depending on the initial values of $a_{i}$, resulting orbits obtained for $h=0.01$ (i.e., sufficiently small value of the sampling interval) and for $t_{0}=0$ have revealed very different asymptotic behavior. Generally, for the initial values (at $n=0)$ of the coefficients $a_{1}$ and $a_{2}$ very close to zero, both these coefficients tend to zero as $n$ increases. This suggests that strong electric fields can establish the uniformity of the molecular orientation distribution across smectic layers in systems with very weak surface anchoring interactions (initial values of $a_{1}$ and $a_{2}$ are then small in 


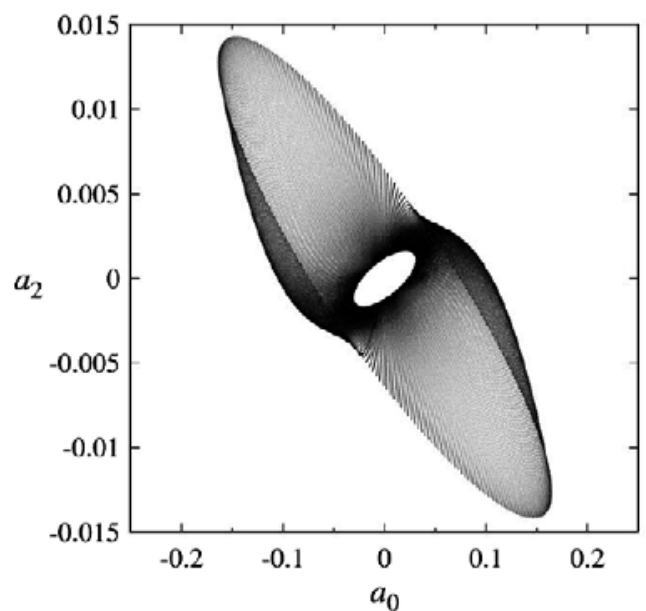

Fig. 3. Projection of the trajectory obtained numerically at $U=20 \mathrm{~V}$ and $f=4 \mathrm{kHz}$, for $0 \leqslant n \leqslant 185000$, starting from $a_{0}^{(0)}=-0.01, a_{1}^{(0)}=0.3$, and $a_{2}^{(0)}=-0.003$.

absolute values). Another case of trivial trajectories takes place if at least one of the coefficients $a_{1}$ and $a_{2}$ is initially large enough in absolute value. Then, the action of the electric field leads to an indefinite enhancement of the level of spatial nonuniformity of the azimuthal angle. In this case, molecules are allowed to perform complete rotations, independently of each other. This seems to be rather unrealistic since in real systems the LC material is bounded by rigid cell plates, and in order to complete rotations would be possible, molecular reorientations should have to some extent cooperative and consistent character. Hence, it follows that a proper description of field-induced nonlinear effects in SSFLCs with very strong surface anchoring of molecules requires taking into account spatial restrictions of molecular motions. There exists, however, a set of immediate initial values of $a_{i}^{(0)}, i=0,1,2$, for which trajectories evolve in a nontrivial, complex way, but are confined to a finite subspace, reflecting partially unsynchronized oscillatory character of molecular motions at high frequencies of strong fields.

Characteristic properties of the complex trajectories are presented below by the example of the evolution of a representative discrete orbit, generated at $U_{0}=20 \mathrm{~V}$ and $f=4 \mathrm{kHz}$, for the initial values $a_{0}^{(0)}=-0.01, a_{1}^{(0)}=0.3$, and $a_{2}^{(0)}=-0.003$. Projection of the resulting initial fragment of the orbit on the $\left(a_{0}, a_{2}\right)$ plane is presented in figs. 3 and 4 at $U_{0}=20 \mathrm{~V}$, for $0 \leqslant n \leqslant 1.85 \times 10^{5}$ and $10^{7} \leqslant n \leqslant 1.06 \times 10^{7}$, respectively. Note that, for the sampling interval assumed here, the period of the field oscillation corresponds approximately to the number $N_{p}=628$ $\left(N_{p} \approx 2 \pi / h\right)$. Accordingly, the shape of the trajectory shown in fig. 3 might suggest that this orbit is periodic or quasi-periodic [33] in a long time scale compared with $N_{p}$. However, as $n$ further increases, the trajectory reveals a tendency to evolve towards different regions of the coefficients space. This is illustrated in fig. 4, where a long fragment $\left(6 \times 10^{5}\right.$ iteration steps $)$ of the trajectory obtained after $10^{7}$ initial iteration steps is shown on the same

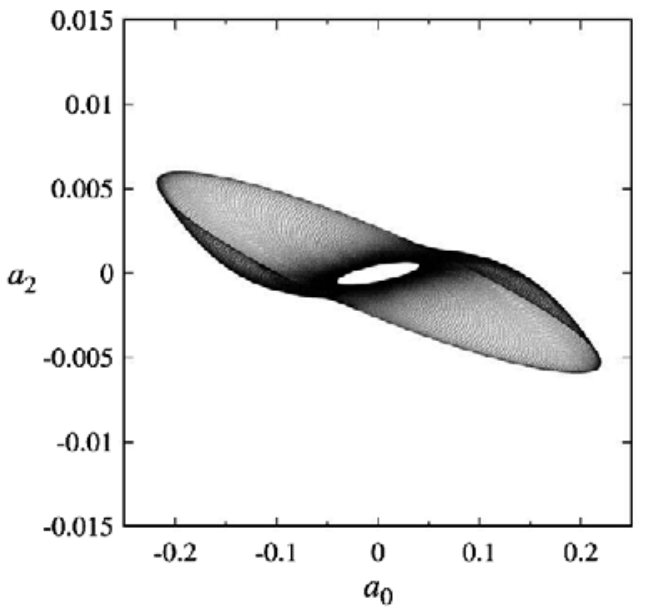

Fig. 4. The trajectory of fig. 3 prolonged for $6 \times 10^{5}$ iteration steps beginning from $n=10^{7}$. Every fifth data point is only plotted.

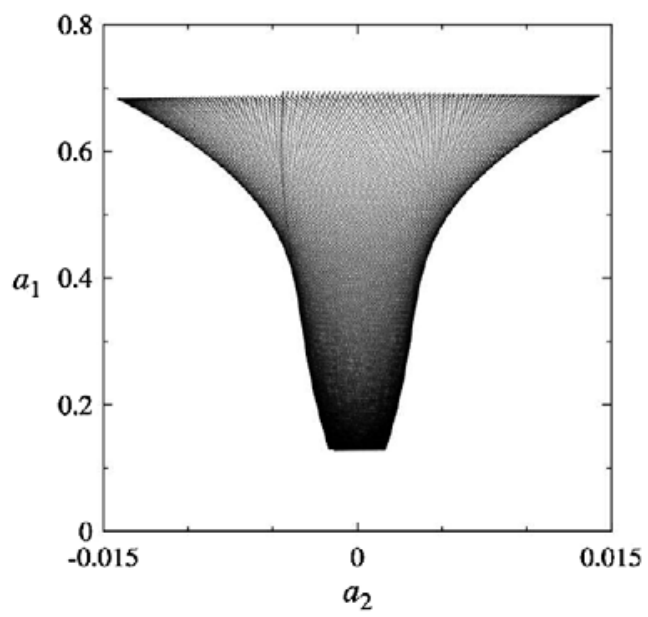

Fig. 5. Projection of the trajectory onto the $\left(a_{2}, a_{1}\right)$ plane, determined at $U=20 \mathrm{~V}$ and $f=4 \mathrm{kHz}$, for $0 \leqslant n \leqslant 185000$, and $a_{0}^{(0)}=-0.01, a_{1}^{(0)}=0.3$, and $a_{2}^{(0)}=-0.003$.

scale as in fig. 3. Such a slow nonperiodic evolution of the trajectory persists over very long time scales. The slow transitory feature of the trajectory is also reflected in its projection onto the $\left(a_{2}, a_{1}\right)$ plane, as shown in figs. 5 and 6 for $U=20 \mathrm{~V}$ and $f=4 \mathrm{kHz}$. The drawings in figs. 3 an 4 exemplify results on systematic analysis of the long-time behavior of the considered orbit. These results seem to indicate that, as $n \rightarrow \infty$, the trajectory ultimately falls into a one-dimensional subspace determined by $a_{1}=0$ and $a_{2}=0$. Then, after an infinite time, the applied alternating electric field would completely suppress the nonuniformity of the space distribution of the azimuthal angle.

Results of figs. $3-6$ evidently show that time dependences of the coefficients $a_{i}, i=0,1,2$, are different. Then, in view of eq. (5), the time evolution of the azimuthal angle $\phi$ differs for particular positions of molecules located within a given smectic layer. This has a direct consequence for the dynamics of the whole cell, as reorientations of 


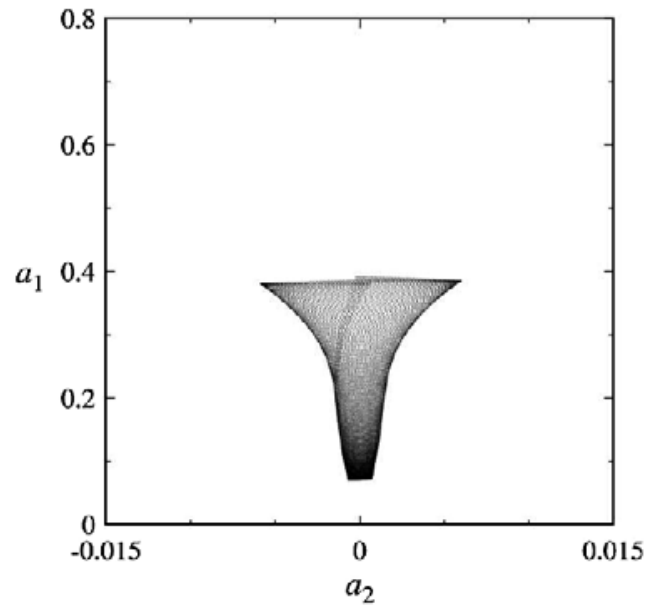

Fig. 6. Prolongation of the trajectory of fig. 5 for $6 \times 10^{5}$ iteration steps starting from $n=10^{7}$. Every 20th data point is plotted.

molecules cannot be considered to be strongly correlated in time. (Even if the correlation of molecular reorientations is initially strong, it becomes weak after a period of time.) Obviously, the complex cell dynamics should be reflected in the response spectra of the system. However, before discussing the effect of the complex dynamics of molecules on dynamic properties of the whole system, it is instructive to analyze the asymptotic behavior of the coefficients $a_{i}$.

To gain more insight into the nature of the longtime effect of external fields on the dynamic behavior of the studied systems, local Lyapunov exponents $\lambda_{i}$, $i=1,2,3,[34-36]$ have been calculated for time series $n=0,1, \ldots, N$ of different (finite) length $N$. Calculations have been carried out using the method developed by Wolf et al. [36]. Results obtained for $10^{5} \leqslant N \leqslant 2 \times 10^{8}$ and different voltage amplitudes, but for the same voltage frequency $f=4 \mathrm{kHz}$ and the same set of initial values of $a_{i}$, $i=1,2,3$, are presented in fig. 7 . It is seen that the temporal dependences of $\lambda_{i}, i=1,2,3$, change significantly as the control parameter $U_{0}$ varies. Nevertheless, longtime behaviors of $\lambda_{i}$ determined for different $U_{0}$ exhibit some qualitative similarities. It is especially remarkable that all three local Lyapunov exponents take on values close to zero, and that at least one of the exponents is positive for sufficiently large finite $N$. After some initial time period, the exponents $\lambda_{i}$ obtained for $U=20 \mathrm{~V}$ and $U=40 \mathrm{~V}$ tend monotonically or nearly monotonically to zero as $N$ grows. (In the case of $U=60 \mathrm{~V}$, asymptotic behaviors of $\lambda_{i}$ appear to be somewhat more complicated.) Since calculations of local Lyapunov exponents are not very reliable for very large $N$ due to computer accuracy limitations [36], the long-time analysis of $\lambda_{i}$ has not been continued for time periods greater than $2 \times 10^{8}$. Therefore, one cannot conclusively determine the type or types of attractors on which the studied trajectories fall as $N$ approaches infinity. However, in view of results of fig. 7, the behavior of these trajectories over long time periods

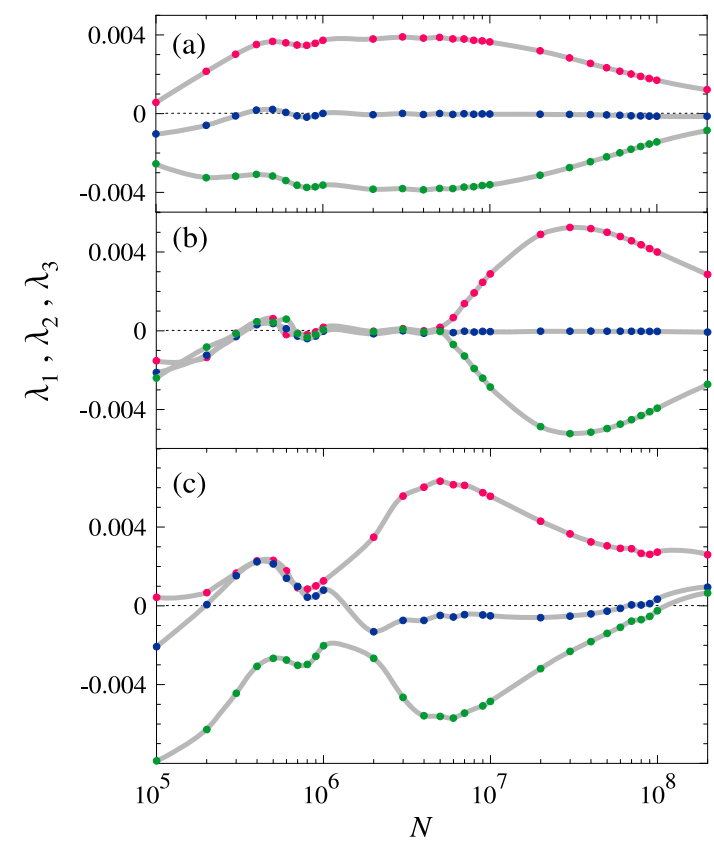

Fig. 7. Local Lyapunov exponents shown as functions of the number of trajectory points generated for $f=4 \mathrm{kHz}$ and $U_{0}=$ $20 \mathrm{~V}(\mathrm{a}), U_{0}=40 \mathrm{~V}(\mathrm{~b})$, and $U_{0}=60 \mathrm{~V}$ (c), starting from the same initial parameter values $a_{0}^{(0)}=-0.01, a_{1}^{(0)}=0.3$, and $a_{2}^{(0)}=-0.003$.

can be classified as long weakly chaotic transients $[37,38]$. During these chaotic transients, the system is very sensitive to even small variations of $U_{0}$ (note that the nonlinearity parameter $c_{2}$ is proportional to $\left.U_{0}\right)$. Then, any changes of $U_{0}$ lead, in general, to unpredictable changes of the dynamic state of the SSFLCs and, thereby, to erratic changes of the shape of high-frequency part of the ColeCole diagram. It should be noted that chaotic behaviors have also been argued to occur in optically excited nematic LCs $[3,4,7-9,15]$. Moreover, the possibility of the occurrence of chaos in smectic LC systems has been reported on the basis of an analysis of dynamic instability that can appear when soliton-like excitations induced by a static external field are perturbed by the application of a slowly oscillating field [39].

Using numerical data for the coefficients $a_{i}$, one can easily determine the azimuthal angle (eq. (5)) as a function of time (at a particular molecular position in a smectic layer). Since the time dependences of $a_{i}, i=1,2,3$, are in general different, the character of the time evolution of $\phi(\tilde{x}, \tilde{t})$ changes as $\tilde{x}$ varies. Although $\phi(\tilde{x}, \tilde{t})$ exhibits fast oscillations which, for all $\tilde{x}$, have the same frequency, equal to the field frequency, the amplitude of these oscillations undergoes a superimposed, nearly periodic modulation of the depth $A_{m}$, which depends on $\tilde{x}$. Furthermore, for small enough $\tilde{x}$, the superimposed phase modulation transits nearly periodically from in-phase mode to antiphase mode and conversely. The aperiodic character of the modulation becomes noticeable for long time periods, much longer than the period of the field alternation. Thus, as far as not too long time periods are considered, the 


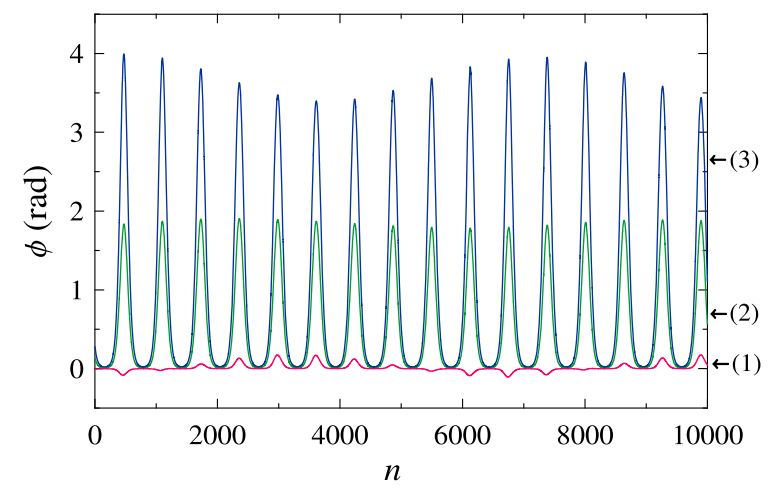

Fig. 8. Time dependence of the azimuthal angle plotted as a function of the number of trajectory points generated for $U_{0}=60 \mathrm{~V}$ and $f=4 \mathrm{kHz}$, starting from the initial parameter values $a_{0}^{(0)}=-0.01, a_{1}^{(0)}=0.3$, and $a_{2}^{(0)}=-0.003: \tilde{x}=0.01$ (1), $\tilde{x}=0.5(2), \tilde{x}=1.0(3)$. Data points are connected by lines.

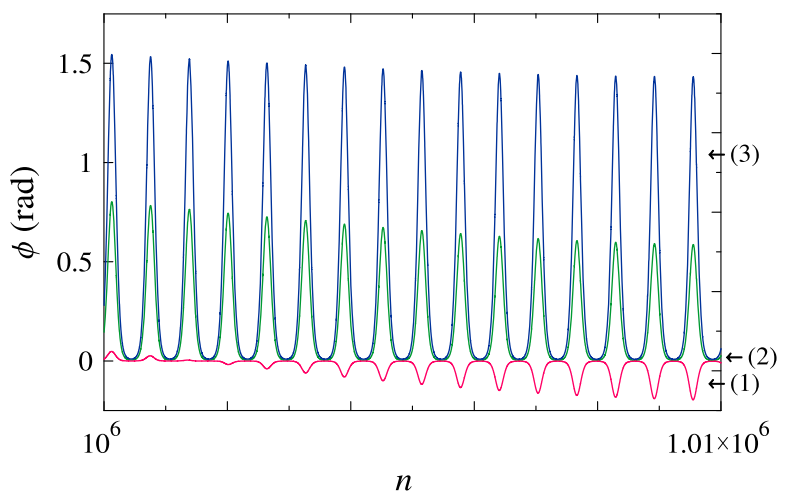

Fig. 9. The plot of fig. 8 , continued through iteration steps $10^{6} \leq n \leq 10^{6}+10^{4}$.

modulation can be treated as being periodic. The quasifrequency $f_{m}$ of such an aperiodic modulation of $\phi(\tilde{x}, \tilde{t})$ is identical for all $\tilde{x}$ and is, in general, much smaller than the field frequency.

It should be also noted that both $A_{m}$ and $f_{m}$ depend on the frequency and the amplitude of applied voltages (for the same time periods). In particular, the frequency $f_{m}$ turns out to be an increasing function of $f$ and a decreasing function of $U_{0}$ (within appropriate ranges of sufficiently large $f$ and $U_{0}$ ). Note that the field-induced modulation of the amplitude of the molecular azimuthal angle is a strongly nonlinear effect that occurs when the space distribution of this angle within smectic layers is inhomogeneous in the absence of the external field and when the space inhomogeneity persists over long periods of time after a strong alternating electric field is turned on. Clearly, space inhomogeneity of SSFLC systems is imposed by anchoring interactions at bounding plates of these systems.

For a better visual presentation, the time dependence of $\phi$ has been shown (figs. 8 and 9) for relatively large $U_{0}=60 \mathrm{~V}\left(A_{m}\right.$ is then very large $)$. In fig. 8, the temporal evolution of $\phi$ is plotted at various molecular positions, for

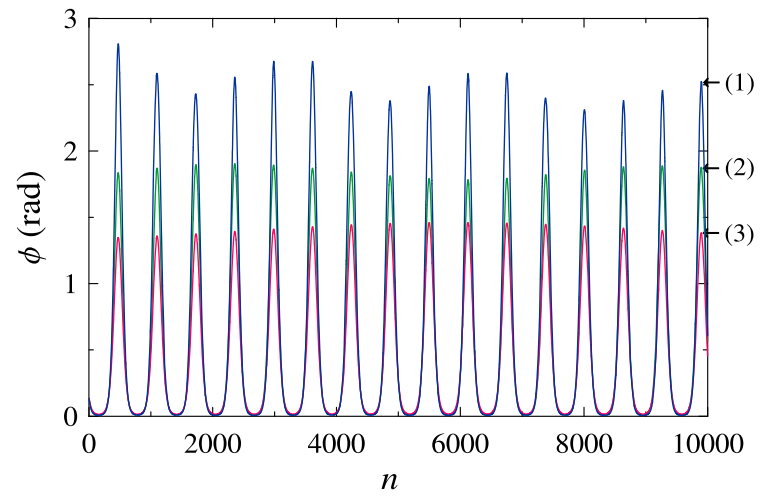

Fig. 10. Time dependence of the azimuthal angle at $\tilde{x}=0.5$ determined for $U=60 \mathrm{~V}$ and different field frequencies: $f=$ $3.5 \mathrm{kHz}(1), f=4.0 \mathrm{kHz}(2)$, and $f=4.5 \mathrm{kHz}$ (3). Initial parameter values were assumed to be $a_{0}^{(0)}=-0.01, a_{1}^{(0)}=0.3$, and $a_{2}^{(0)}=-0.003$.

initial $N=10^{4}$ iteration steps, while a further evolution, after $10^{7}$ iterative steps, is drawn in fig. 9 for the same positions of molecules and for the same time period. It is seen that $\phi$ exhibits an asymmetric behavior near boundary $\operatorname{surfaces}(i . e .$, for $\tilde{x} \approx 0$ and $\tilde{x} \approx 1$ ). Obviously, such a asymmetry is a consequence of specific boundary conditions assumed here to reflect the asymmetry of surface anchoring interactions in typical SSFLCs [30,31]. Comparing the drawings of fig. 8 and 9 evidently shows that $f_{m}$ becomes smaller as the number of iterative steps grows. In spite of that, the modulation frequency appears to be identical for all $\tilde{x}$ (in the same relatively short time periods), the modulation depth $A_{m}$ is rather strongly space dependent. Moreover, the phase shift $\beta_{m}$ between modulations of $\phi(\tilde{x}+\delta \tilde{x}, \tilde{t})$, where $\delta \tilde{x} \neq 0$, such that $0 \leqslant \tilde{x}+\delta \tilde{x} \leqslant 1$, and $\phi(\tilde{x}, \tilde{t})$ is nonzero and also depends on $\tilde{x}$. This is in contrast with the corresponding phase shift $\beta_{f}$ between fast oscillations (with the frequency $f$ ) at different molecular positions. As seen in figs. 8 and $9, \beta_{f}$ takes only two values: 0 or $\pi$.

The space and time dependence of the depth modulation of fast oscillations (with the frequency $f$ ) of the azimuthal angle as well as the space variation of phase of modulations are a clear sign of desynchronization of molecular reorientations. The complexity of these processes is also reflected in a rather strong dependence (for fixed $U_{0}$ and $\tilde{x}$ ) of $f_{m}, A_{m}$, and $\beta_{m}$ on the field frequency, as illustrated in fig. 10. Since $f_{m}<f$, the modulation of the amplitude of molecular reorientations occurs in a longer time scale than the temporal scale of the field oscillations. Nevertheless, the $f$-dependence of $f_{m}$, and thereby the $f$-dependence of $A_{m}$ and $\beta$ leads to an intricate variation of the azimuthal angle when $f$ is changed, and, consequently, leads to a complex form of the response spec$\operatorname{tra} \varepsilon(\omega)$ like those shown in fig. 1. The reason for the occurrence of a high-frequency band in these spectra is the nonuniformity of the depth and the phase shift of the modulation. It should be pointed out that, although the phase of fast oscillatory molecular reorientations is fully 
synchronized, the phase of quasi-periodic modulation, and thereby the overall reorientation motion of molecules, is not synchronized (due to the nonuniformity of the amplitude and phase of the modulation). The nonuniformity of molecular reorientations can be considered as the existence of small regions (with respect to the thickness of a sample) in smectic layers, within which molecules can undergo nearly synchronized reorientation motions with specific relaxation times shorter than the relaxation time of Goldstone-mode oscillations. However, to show this explicitly, one would know the functional form of the contribution to $\varepsilon(\omega)$ coming from local changes of $\phi$ under strong fields.

It must be stressed that, although the theoretical model considered here predicts, for fixed $U_{0}$ and $f$, the occurrence of a single quasi-frequency $f_{m}$ of the modulation of fast oscillatory molecular reorientations, one can expect that the nonlinear response of real SSFLC systems displays the existence of a broad low-frequency band rather than a single signal (at the frequency $f_{m}<f$ ). Indeed, the modulation of reorientation oscillations of molecules appears when SSFLCs remain in the chaotic state during long time periods. Then, the time evolution of the systems can strongly be influenced even by small fluctuations of parameters controlling the nonlinear behavior of the systems [38]. In particular, such fluctuations can originate in device noise, associated, e.g., with uncontrollable variations of the amplitude and/or frequency of the applied field. Clearly, the resulting perturbation of the time evolution of SSFLCs can lead to a considerable smearing of signals registered at frequencies that are lower than the field frequency.

There emerges the question if a more adequate description of the complex nonlinear behavior of SSFLCs would require a generalization of the motion equation to include depolarization and anisotropy contributions [9]. Since both these contributions exhibit the same dependence on the azimuthal angle and the depolarization contribution is usually much greater (in absolute value) than the anisotropy one $[23,24]$, the latter contribution can be neglected. As concerns the depolarization, this process is responsible for excitation of local soliton-like excitations propagating within smectic layers [22], but is rather of minor importance for establishing complex nonlocal, weakly correlated molecular motions. This follows from the fact that the depolarization process is significant in SSFLCs when the azimuthal angle is strongly inhomogeneous, i.e. when at least one of the parameters $a_{1}, a_{2}$ is sufficiently large in absolute value. In that case, most of molecules would perform large-amplitude oscillations, or even full rotations, nearly independent of each other. As such inconsistent large-amplitude reorientations of molecules are suppressed in real systems by rigid cell plates and couplings between molecules belonging to adjacent smectic layers, the level of inhomogeneity of the azimuthal angle through entire smectic layers cannot be very large. Consequently, the depolarization effect can also be omitted in describing complex, partially correlated molecular reorientations of not too large amplitudes. Numerical results obtained for different $U_{0}$ show that the amplitude of $\phi$ is not very large even for relatively high voltages (say, for $U_{0}$ not greater than $40 \mathrm{~V}$ ), and systematically decreases with time.

It is obvious that the mere modulation of $\phi$ should be reflected in the electro-optic response of SSFLCs at frequencies lower than the current frequency of the applied field. Since chaotic trajectories are very sensitive even on weak external noise [33], one should expect that response signals associated with the modulation of fast oscillations of molecular orientations will appear in real systems over some frequency range rather than at a rigidly determined modulation frequency. This does not mean, however, that external noise (coming, e.g., from the measuring device) destroys possible long-lived chaotic transient states of the studied systems. Indeed, a typical property of chaotic transients is their stability against external noise [40]. In the next section, an approach to detect the electro-optic response at frequencies below the frequency of an applied field is presented.

\section{Experimental evidence of low-frequency nonlinear effects}

As shown in the preceding section, the application of strong electric fields of sufficiently high frequencies to a ferroelectric LC sample causes complex reorientational motions of molecules. This gives rise to corresponding changes in optical transmission of the sample placed between crossed polarizers and, thereby, leads to the occurrence of the electro-optic effect. Then, using the photodiode light detector, the transmittance changes can be converted into appropriate variations in the output voltage, easy to register. If the field is strong enough to produce nonlinear effects, the amplitude of oscillations of transmitted light intensity is expected to vary as described above, i.e., the amplitude of oscillation of the intensity of transmitted light is expected to undergo a quasi-periodic modulation that might be detectable at frequencies lower than the excitation (field) frequency $f$. However, the depth of this modulation can be small but a component of frequency $f_{m}$ could be measurable, if the sensitivity of detection system was sufficiently high. Accordingly, a simple method to enhance the sensitivity (i.e., the signal/noise ratio) consists in narrowing the frequency window of the detection circuitry. In our experiment, this requirement was fulfilled by using a selective amplifier of the type 233 made by Unipan (Warsaw). This instrument has a sensitivity better than $10^{-7} \mathrm{~V}$ rms and possesses adjustable selectivity up to $54 \mathrm{~dB}$ per octave. Such a selectivity is equivalent to the FWHM (full with at half-maximum) of about $8 \%$ in the frequency scale. The center of the transmitted frequency is continuously tunable from $1.5 \mathrm{~Hz}$ to $150 \mathrm{kHz}$. The amplitude of voltages used in experiments was changed from a fraction of a volt up to $20 \mathrm{~V}$ (rms), while the voltage frequency was varied in the range from $1 \mathrm{kHz}$ to $10 \mathrm{kHz}$. The modulation components were measured at frequencies ranging from $1.5 \mathrm{~Hz}$ to frequencies above the field frequency. A scheme of the measuring setup is presented in fig. 11 . 


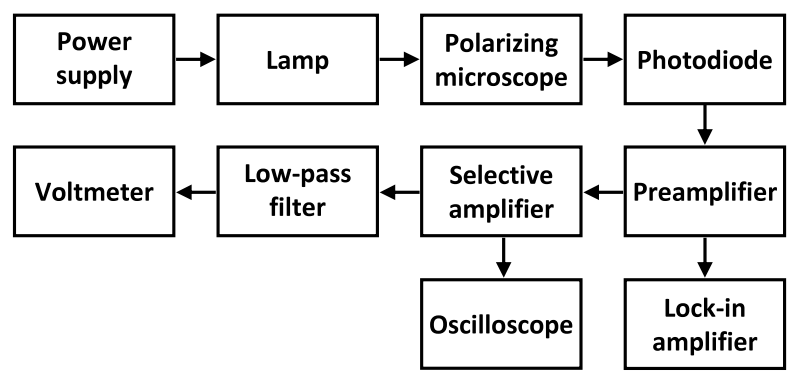

Fig. 11. Simplified scheme of the experimental setup.

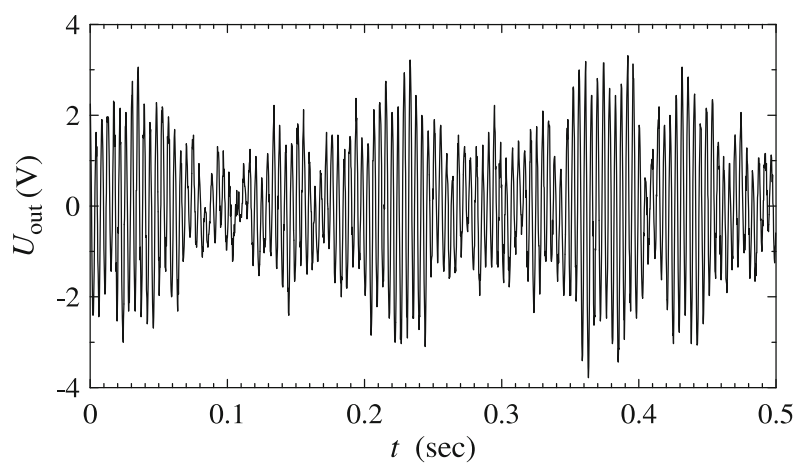

Fig. 12. Typical oscilloscope picture of fluctuations of the amplified selective voltmeter output obtained for a sample of Felix 17-100. The amplitude and frequency of the applied voltage were, respectively, $U_{0}=20 \mathrm{~V}$ and $f=4 \mathrm{kHz}$. The selective voltmeter was set to the frequency $f_{s}=230 \mathrm{~Hz}$.

Obviously, the modulation of the transmittance oscillations has been searched at frequencies lower than the frequency of applied external field. Despite careful search we did not succeed in finding any modulation of stable frequency (except for the frequency of the electric power and its harmonics, which had no interest here). Instead, we observed large fluctuations of the amplified output voltage $U_{\text {out }}$ of the selective amplifier at different frequencies $f_{s}$ adjusted for the transmission. Note that $U_{\text {out }}$, measured at selected fixed frequencies $f_{s}$ less than the field frequency, characterizes possible modulations of fast transmittance oscillations (these oscillations proceed with the field frequency). Exemplary time variation of $U_{\text {out }}$, registered for the selected frequency $f_{s}=230 \mathrm{~Hz}$, is shown in fig. 12 . This figure indeed displays the existence of a relatively slow modulation of light transmittance oscillations (the modulation is visible in the figure as quick periodic variations of the period $\left.T_{s}=(230 \mathrm{~Hz})^{-1} \approx 0.004 \mathrm{~s}\right)$. However, the amplitude of the modulation behaves very irregularly in time. It is evident from fig. 12 that, although registered signals exhibit modulated fast oscillations, the modulation cannot be characterized by a single frequency or even by a single quasi-frequency. As already mentioned in the preceding section, such an irregularity in the modulation can be considered as resulting from a large sensitiveness of the studied strongly nonlinear systems to the external noise. Next, instant absolute values of $U_{\text {out }}$ were averaged using a low-pas RC filter with the time constant

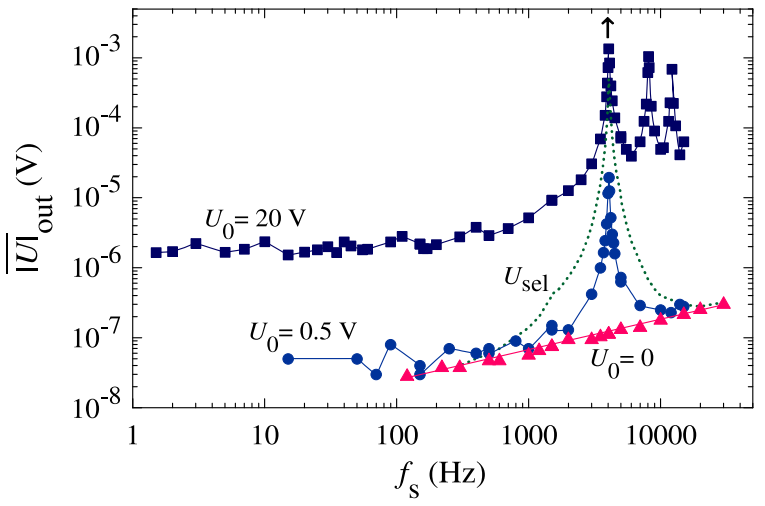

Fig. 13. Dependence of the average amplitude of the voltage at the output of selective voltmeter on the selected frequency (corresponding to the modulation frequency), derived for the Felix 17-100 mixture at the applied voltage of the frequency $f=4 \mathrm{kHz}$ (indicated by an arrow) and of different amplitudes: $U_{0}=0(\boldsymbol{\Delta}), U_{0}=0.5 \mathrm{~V}(\bullet)$, and $U_{0}=20 \mathrm{~V}(\boldsymbol{\square})$. For comparison, the selectivity curve of the amplifier is presented (dotted line). This curve has been obtained by applying the voltage of the appropriately adjusted amplitude $U_{\text {sel }}=500 \mu \mathrm{V}$ and frequency $4 \mathrm{kHz}$ directly to the amplifier input (see the text).

of several seconds. Results for the averaged output voltage $\overline{|U|}_{\text {out }}$ obtained at the excitation frequency $f=4 \mathrm{kHz}$ are illustrated in fig. 13 for different amplitudes of the applied voltage. A strong response of the system at the excitation frequency is seen. In the case of high voltage $\left(U_{0}=20 \mathrm{~V}\right)$, a distinct response occurs also at the second and third harmonics of the excitation frequency. More interesting is, however, the existence of a wide low-frequency band in the nonlinear response spectrum. Comparing lowfrequency spectra derived for $U_{0}=0.5 \mathrm{~V}$ and $U_{0}=0$ indicates that the low-frequency band is absent in the case of linear spectra. In view of the analysis of the previous section, the existence of the low-frequency band reflects the occurrence of modulations of molecular reorientational oscillations, which proceed with the field frequency. The appearance of a wide low-frequency band (rather than a single peak corresponding to a definite modulation frequency $\left.f_{m}\right)$ in nonlinear response spectra, represented here by the dependence of $\overline{|U|}_{\text {out }}$ on the selected frequency $f_{s}$, can be attributed to a field-provoked large sensitivity of the studied systems on the external noise. To convince oneself that the low-frequency band in the nonlinear spectrum $\overline{|U|}_{\text {out }}\left(f_{s}\right)$ is not generated by the very selective amplifier as a consequence of its finite selectivity, the selectivity spectrum of the amplifier was determined by applying a voltage of frequency $4 \mathrm{kHz}$ directly to the input of the amplifier. The amplitude of this voltage was taken approximately the same as the amplitude of the preamplifier output (see fig. 11), resulting from molecular reorientations under an external voltage of the amplitude $U_{0}=20 \mathrm{~V}$ and of the frequency $f=4 \mathrm{kHz}$. Then, at $U_{0}=20 \mathrm{~V}$ and $f_{s}=4 \mathrm{kHz}$, the averaged output voltage $\overline{|U|_{\text {out }}}$ had the same magnitude for signals originating both in molecular reorientations induced by external alternating voltage and 
in alternations of the voltage applied to the amplifier input (see fig. 11). However, within a wide range of selected frequencies below $4 \mathrm{kHz}$, the response spectrum obtained for $U_{\text {sel }}$ is much lower than the nonlinear spectrum (determined for $U_{0}=20 \mathrm{~V}$ ), and approximately coincides with the linear spectrum (derived for $U_{0}=0.5 \mathrm{~V}$ ). Thus, the finite selectivity of amplifier is not responsible for the appearance of the low-frequency band in the nonlinear spectra $\overline{|U|}_{\text {out }}\left(f_{s}\right)$.

The selective measurement of the field-induced response of LCs at a chosen frequency can, in principle, be performed by applying phase detection technique using the lock-in amplifier. We tried to apply this technique but the attempt was, however, unsuccessful. We suppose that this was caused by the extremely narrow frequency window, amounting to a small fraction of a hertz, when the lock-in amplifier is working in its standard mode. As well known, it is extremely difficult to synchronize the phase detector with a signal of unknown frequency with the precision of a fraction of one hertz. Moreover, it can be expected that the fluctuations of modulation frequency $f_{m}$ are much larger than the frequency window of the detector. Then, the synchronization will never be finished, because the process of synchronization of the detector needs a sufficiently long time period. Clearly, the frequency window of the detector must be small (to reduce noises) but not too small, so as to allow the synchronization process to be completed. This requirement makes the application of the lock-in amplifier to selective measurements rather aimless. There emerges, however, another opportunity to employ the lock-in amplifier in investigating low-frequency contributions to response spectra of the studied systems.

As seen in fig. 12, the amplitude of oscillations (with the frequency $f_{s}$ ) of the voltage $U_{\text {out }}$ strongly fluctuates in time. Thus, the depth of modulation of fast transmittance oscillations behaves as it would undergo an intense noise. Consequently, one can try to detect low-frequency modulations of oscillatory alternations of the transmittance using methods originally developed for noise measurements, i.e., one can attempt to detect these modulations through measuring the level of noise of registering signals. For this purpose, we applied the lock-in amplifier of the type SR 530 (Stanford Research). Furthermore, to ascertain whether the modulation effect is not specific only to the Felix 17-100 LC material, noise measurements were carried out for samples containing the Felix 15-100 mixture, in the same conditions and within the same range of selected frequencies as in the experiment described above. The frequency window was set equal to $10 \mathrm{~Hz}$. Results of measurements are presented in fig. 14, where the level of noise is represented by the average of absolute value $\overline{|U|}_{\mathrm{n}}$ of the voltage $U_{\mathrm{n}}$ at the output of the lock-in amplifier. The similarity of these results to those obtained using the selective amplifier is evident (cf., fig. 13). The noise measurements also reveal the occurrence of the lowfrequency band (associated with the modulation of fast oscillations) in the nonlinear response spectra (obtained for $\left.U_{0}=20 \mathrm{~V}\right)$. For $U_{0}=0.5 \mathrm{~V}$, the modulation with the frequency less than $1 \mathrm{kHz}$ is not detectable, as in the

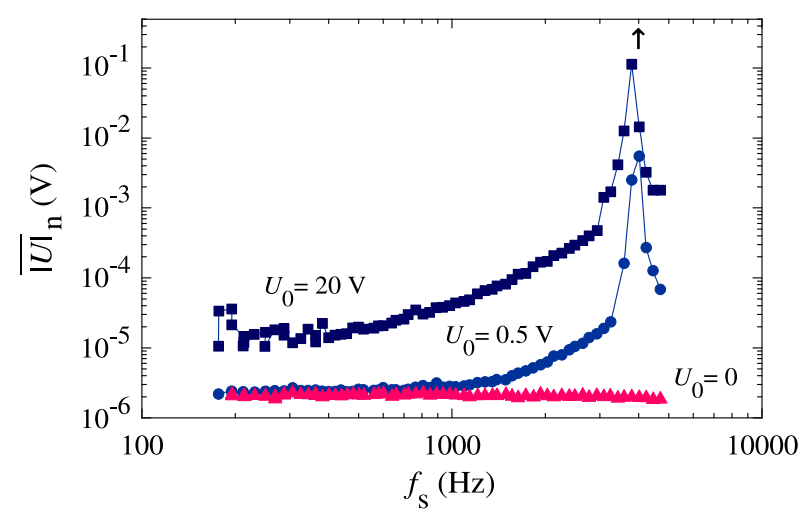

Fig. 14. Frequency dependence of the noise level registered for the Felix 15-100 LC as the average amplitude of voltage in the output of the lock-in amplifier SR 530, at the same frequency $f=4 \mathrm{kHz}$ but at different amplitudes: $U_{0}=0(\boldsymbol{\Delta}), U_{0}=0.5 \mathrm{~V}$ $(\bullet)$, and $U_{0}=20 \mathrm{~V}(\boldsymbol{\square})$ of the applied voltage.

case of results of fig. 13. Moreover, the results of fig. 14 indicate that signals registered at $U_{0}=20 \mathrm{~V}$ are much stronger than those measured at $U_{0}=0$. This means that the appearance of the low-frequency band in the nonlinear response spectra cannot be considered as coming from a noise generated by measuring devices used in our experiment. In particular, the photonic noise cannot be considered responsible for the observed dynamic effect, as the intensity of light falling on the photodetector has not changed after applying external voltages (this has been thoroughly checked). Then, experimental results obtained by applying both detection methods give a strong evidence for the existence of relatively slow modulations of light intensity oscillations. Furthermore, the nonlinear character of these modulations has undoubtedly been confirmed.

An important question that arises is whether dynamic processes induced by strong fields of low frequencies have also transitory chaotic, or simply chaotic, character. As already mentioned (sect. 2), nonlinear processes activated at low field frequencies (motions of structural defects, changes in the texture, nonlinear ionic currents, etc.) are much more complex than nonlinear molecular reorientations, investigated here in the case of high-field frequencies. It is rather clear that it would be very difficult to formulate a formalism that would adequately describe complex low-frequency processes and possible interferences between them. Therefore, there is no actual theoretically grounded evidence on whether or not strongly nonlinear low-frequency processes reveal chaotic character. Nevertheless, Cole-Cole diagrams determined on the basis of experimental electro-optic data (fig. 2) reveal a complicated nonmonotonic variation under changes of the external field also within the low-frequency region. It should noted that the complex nonlinear behavior of the studied system has been observed both at weaker and stronger voltage amplitudes than $U_{0}=20 \mathrm{~V}$, used in all experiments as a standard voltage amplitude. However, the lowest value of $U_{0}$ at which the strongly nonlinear behavior of the system can experimentally be registered is not easy to determine in a unique way. 


\section{Conclusions}

Nonlinear dynamic effects induced by strong external alternating electric fields in SSFLCs have been described using the balance torque equation. Numerical solutions of this equation have displayed that strong enough fields of appropriately high frequencies can disorganize correlated molecular reorientations, preferred at weak fields. The resulting complex partially asynchronous orientational motion of molecules has been argued to manifest itself in a long-time scale associated with relatively slow modulations of fast oscillations executed with the field frequency. The depth and the phase shift of these modulations turn out to depend not only on space but also on the frequency of the applied field. This can be responsible for the complex form of the electro-optic spectra determined for strong fields. The effect of modulations of the azimuthal angle has experimentally been detected by registering the electro-optic response of SSFLCs for frequencies lower than the field frequency. Finally, one must point out that the investigation of complex nonlinear dynamics of SSFLCs may be of interest in better understanding the switching processes between stable orientational states when the action of the applied field is very strong, but not strong enough to completely dominate the couplings between molecules forming smectic layers.

Open Access This is an open access article distributed under the terms of the Creative Commons Attribution License (http://creativecommons.org/licenses/by/3.0), which permits unrestricted use, distribution, and reproduction in any medium, provided the original work is properly cited.

\section{References}

1. J.R. Lalanne, J. Buchert, S. Kielich, in Modern Nonlinear Optics, Part 1, edited by M. Evans, S. Kielich (Wiley, New York, 1993).

2. M. Buscalia, T. Bellini, V. Degiorgio, F. Mantegazza, F. Simoni, Europhys. Lett. 48, 634 (1999).

3. G. Russo, V. Carbone, G. Cipparrone, Phys. Rev. E 62 $5036(2000)$.

4. G. Cipparrone, G. Russo, C. Versace, G. Strangi, V. Carbone, Opt. Commun. 173, 1 (2000).

5. Y. Kimura, H. Isono, R. Hayakawa, Eur. Phys. J. E 9, 3 (2002).

6. S.M. Morris, A.D. Ford, M.N. Pivnenko, H.J. Coles, J. Opt. A 7, 215 (2005).

7. B. Piccirillo, A. Vella, A. Setaro, E. Santamato, Phys. Rev. E 73, 062701 (2006).

8. L. Lucchetti, M. Gentili, F. Simoni, S. Pavliuchenko, S. Subota, V. Reshetnyak, Phys. Rev. E 78, 061706 (2008).

9. S.T. Lagerwall, Ferroelectric and Antiferroelectric Liquid Crystals (Wiley-VCH, Weinheim, 1999).
10. L. Lam, Z.C. Ou-Yang, M. Lax, Phys. Rev. A 37, 3469 (1988).

11. H. Orihara, A. Fukase, S. Izumi, Y. Ishibashi, Ferroelectrics 147, 411 (1993).

12. Y. Ishibashi, H. Orihara, Frerroelectrics 156, 185 (1994).

13. H. Orihara, Y. Isibashi, J. Phys. Soc. Jpn. 64, 3775 (1995).

14. Y. Ishibashi, H. Orihara, Physica B 219-220, 626 (1996).

15. G. Demeter, L. Kramer, Phys. Rev. Lett. 83, 4744 (1999).

16. Y. Kimura, S. Hara, R. Hayakawa, Ferroelectrics 245, 61 (2000).

17. Y. Kimura, S. Hara, R. Hayakawa, Phys. Rev. E 62, R5907 (2000).

18. T.D. Frank, Phys. Rev. E 72, 041703 (2005).

19. J.M. Leblond, R. Douali, C. Legrand, R. Dạbrowski, Eur. Phys. J. Appl. Phys. 36, 157 (2006).

20. C. Thibierge, D. L'ôte, F. Ladieu, R. Tourbot, Rev. Sci. Instrum. 79, 103905 (2008).

21. N.A. Clark, S.T. Lagerwall, in Ferroelectric Liquid Crystals, Principles, Properties and Applications, edited by J.W. Goodbye, R. Blinc, N.A. Clark, S.T. Lagerwall, M.A. Osipov, S.A. Pikin, T. Sakurai, K. Yoshino, B. Žekš (Gordon and Breach, Philadelphia, 1991).

22. W. Jeżewski, W. Kuczyński, J. Hoffmann, Phys. Rev. E 83, 042701 (2011).

23. R.E. Amritkar, N. Gupte, Phys. Rev. A 44, R3403 (1991).

24. M.D. LaMar, G.D. Smith, Phys. Rev. E 2010 81, 046206 (2010).

25. W. Kuczyński, J. Hoffmann, J. Małecki, Ferroelectrics 150, 279 (1993).

26. W. Jeżewski, W. Kuczyński, J. Hoffmann, Liq. Cryst. 34, 1299 (2007)

27. W. Jeżewski, W. Kuczyński, J. Hoffmann, Phys. Rev. E 73, $061702(2006)$.

28. W. Jeżewski, W. Kuczyński, J. Hoffmann, Phys. Rev. B 77, 094101 (2008).

29. W. Jeżewski, W. Kuczyński, Phys. Rev. B 79, 214206 (2009).

30. M.A. Handschy, N.A. Clark, S.T. Lagerwall, Phys. Rev. Lett. 51, 471 (1983).

31. J.E. Maclennan, M.A. Handschy, N.A. Clark, Liq. Cryst. 7, 787 (1990).

32. P.J. Davies, I. Polonsky, in Handbook of Mathematical Functions with Formulas, Graphs, and Mathematical Tables, edited by M. Abramowitz, I.A. Stegun (Dover, New York, 1972) p. 896.

33. E. Ott, Chaos in Dynamical Systems (Cambridge University Press, New York, 1993).

34. R.C.L. Wolf, J. R. Stat. Soc. B 54, 353 (1992).

35. F. Ali, M. Menzinger, Chaos 9, 348 (1999).

36. A. Wolf, J.B. Swift, H.L. Swinney, J.A. Vastano, Physica D 16, 285 (1985).

37. G.M. Zaslavsky, R.Z. Sagdeev, D.A. Usikov, A.A. Chernikov, Weak Chaos and Quasi-Regular Patterns (Cambridge University Press, Cambridge, 1991).

38. R.C. Hilborn, Chaos and Nonlinear Dynamics (Oxford University Press, Oxford, 2000).

39. I.W. Stewart, T. Carlsson, F.M. Leslie, Ferroelectrics 148, 41 (1993).

40. H. Katz, P. Grassberger, Physica D 17, 75 (1985). 